\title{
Optimization of parameters of the electromechanical converter with a discrete secondary part
}

\author{
Denis Kuimov ${ }^{1, *}$, Alexandr Pavlenko², Maksim Minkin ${ }^{1}$ \\ ${ }^{1}$ Don State Technical University, Chair "Production Process Automation”, 344000 Rostov-on-Don \\ Gagarin square 1, Russia \\ ${ }^{2}$ Southern Russian state polytechnical university (NPI) of M.I. Platov. Russia, 346428 Novocherkassk \\ Prosveshcheniya St. 132, Russia
}

\begin{abstract}
The algorithm of multicriteria optimization of a tooth zone of the electromechanical converter with a discrete secondary part realized on the basis of application of the program Ansys complex is offered. The optimum geometry of a tooth zone of the inductor of the electromechanical converter with a discrete secondary part on condition of achievement of the greatest possible value of the electromagnetic effort operating on ferromagnetic elements is defined. As a method of optimization the stochastic Monte Carlo method has been used.
\end{abstract}

\section{Introduction}

The electromechanical converter with a discrete secondary part (ECDSP) the perspective device used in technological processing of liquid materials [1-3], including oil products [4-6]. A basic element of ECDSP is the unique working body representing set of the bodies of ferromagnetic material making a secondary discrete part and making the difficult movement under the influence of the external electromagnetic field. The movement of ferromagnetic elements generates in the processed liquid raw materials a set of various power influences from usual impacts and mechanical friction, before hydrodynamic cavitation, thereby creating the big density of energy per substance unit of volume.

At the expense of the unique opportunities in processing of liquid environments in the near future ECDSP and devices similar to him, can make the serious competition to traditional mechanical mixers, colloidal mills and another, devices similar to destination in many industries.

The design of ECDSP [7] offered earlier by authors of article which sketch is submitted in fig. 1 is rather simple and reliable, has 4 basic elements, including the cylindrical inductor, the concentrated winding, the working camera made of not magnetic material and located in boring of the inductor a set of the ferromagnetic elements representing a secondary discrete part.

\footnotetext{
* Corresponding author: kuimov_d@list.ru
} 


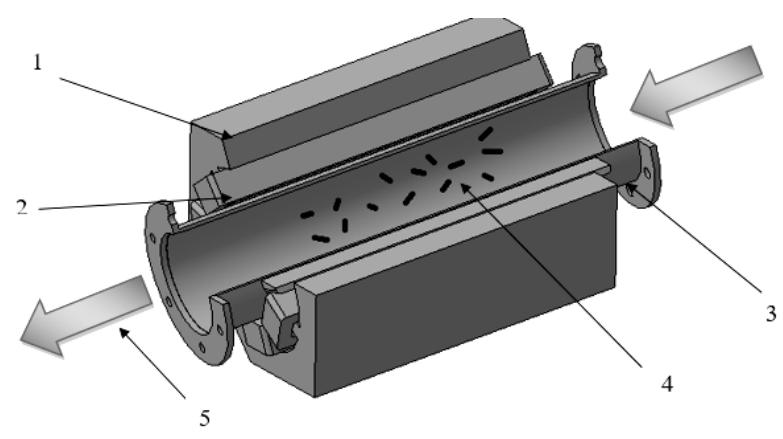

Fig. 1. Standard design of the electromechanical converter with a secondary mobile part:

1 -inductor; 2 - the 3 phase distributed winding; 3 - a pipe from not magnetic material; 4 - set of ferromagnetic elements; 5 - a stream of the processed raw materials.

At the same time ECDSP has shortcomings, sharply non-uniform electromagnetic field caused by a big gap and lack of the monolithic magnetic core, generating "dead" zones of the 1st and 2nd order is basic of which.

The main stage of creation of the electromechanical converter, his design calculation is. At design of the previous devices of this kind the technique based first of all on achievement of average value of magnetic induction on the volume of the working camera in the range of 0,15-0,2 $\mathrm{T}$ was applied. However for design of energy efficient installation the analysis of distribution of the electromagnetic field on the working camera doesn't suffice. A relevant task is the problem of synthesis of magnetic system of the electromechanical converter. Results of design of ECDSP are given below.

\section{Main part}

The quality of work of the projected ECDSP first of all is defined by quantity of the working ferromagnetic elements involved in the general movement. The chosen configuration of the inductor has to provide such distribution of the electromagnetic field in a working zone at which perhaps full elimination of "dead" zones of the 1 st and 2nd order. Problem definition of optimization of a tooth zone of the inductor can be considered correct only in that case when criteria and parameters of optimization are specified and proved, in other words as function of the purpose and function of restrictions is formulated [8].

The inductor ECDSP design at the same time has to meet several requirements, many of which can be contradictory. For example, providing easier conditions of elimination of "dead" zones demands reduction of internal radius of the inductor that in turn reduces the mass of the inductor, reduces winding volume, but at the same time leads to essential reduction of bandwidth of the device. On the other hand increase in MDS of a winding for increase in the electromagnetic effort operating on a working ferromagnetic element leads to increase in power consumption and increase in volume of a winding. In this regard the combined approach to the solution of a problem of optimization has been chosen $[8,9]$. As the geometrical sizes of the inductor of the device act as the optimized parameters, optimization is parametrical [10].

For the solution of a problem of optimization of the inductor ECDSP first of all it is necessary to decide on the criteria of optimality which are function from optimization parameters. As parameters of optimization the geometrical sizes of tooth zone ECDSP (fig. 2) were accepted: inductor b1 tooth crown height; inductor b2 tooth width; b3 tooth crown width. 


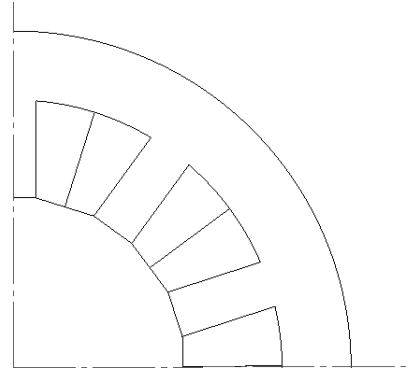

Fig. 2. Cross-section of initial geometry of a tooth zone of the inductor of the device.

For these parameters, mathematical expression of criterion function can be written down. The efficiency of work of the electromechanical converter with a discrete secondary part is estimated first of all by efficiency of the movement of ferromagnetic elements on the working camera which in turn is determined by the nature of distribution of magnetic field by the working camera when the average value of magnetic induction on the volume of the working camera reaches values of 0,15-0,2 $\mathrm{T}$ [2]. However often achievement of a present value of magnetic induction doesn't provide the optimum movement of secondary elements that is confirmed by the results presented in [11]. Therefore as key parameter the average electromagnetic effort influencing from the created electromagnetic field a single ferromagnetic working element on all volume of the working camera is chosen. A condition of restriction of admissible heating of steel of the inductor and, respectively, the condition of restriction of magnetic induction in a magnetic conductor will be the additional parameters of optimization acting as functions of restrictions. Thus, most good results by optimization of a tooth zone of the inductor of the electromechanical converter with a discrete secondary part can be achieved, only using multicomponent criterion function of the following look:

$$
F_{u}=k_{1}\left(\frac{F_{m}-F_{m T}}{F_{m}}\right)+k_{2}\left(\frac{B-B_{T}}{B}\right)+k_{3}\left(\frac{t-t_{T}}{t}\right)+k_{4}\left(\frac{B_{z}-B_{z T}}{B_{z}}\right) \rightarrow \min ,
$$

where $k_{1}, k_{2}, k_{3}, k_{4}$ - weight coefficients for each private criterion of optimization; $F_{m}$ - target average value of the electromagnetic effort operating on a single ferromagnetic element from the electromagnetic field, $\mathrm{N} ; F_{m T}$ - the current average value of the electromagnetic effort operating on a single ferromagnetic element from the electromagnetic field, $\mathrm{N} ; B$ - the target relation of values of magnetic induction on the volume of the working camera, T; $B_{T}$ - the current relation of values of magnetic induction on the volume of the working camera, $\mathrm{T} ; t-$ target value of temperature of steel of a magnetic conductor, ${ }^{\circ} \mathrm{C} ; t_{T}$ - the current value of temperature of a magnetic conductor, ${ }^{\circ} \mathrm{C}$; $B_{z}$ - target value of magnetic induction in steel of a magnetic conductor, T; $B_{z T}$ - the current value of magnetic induction in steel of a magnetic conductor, $\mathrm{T}$.

Values of weight coefficients are chosen depending on degree of the importance of a separate component of criterion of optimization. The sum of weight coefficients always has to equal 1.

Except a task of criterion function, it is necessary to choose the method allowing to pick up the optimum geometrical sizes of a tooth zone of the inductor of the electromechanical converter for rather small period. Analysing scientific works on a subject of optimization $[9,10]$ and also experience of design and development of inductors of electromechanical converters, among a large number of algorithms of optimization, it is established that good results at achievement of a goal can be received with application of a stochastic algorithm 
of optimization of Monte Carlo (a method of random numbers). The essence of the Monte Carlo method consists in search of a required extremum of criterion function by the casual choice of the geometrical parameters defining the magnetic system of the inductor of the electromechanical converter. In practice this method means carrying out $\mathrm{N}$-go of number of tests as a result of which receive $\mathrm{N}$ possible values of required function. On the basis of it the conclusion about a high error of the chosen method which size is inversely proportional to the number of tests [9] is drawn. From this it follows that the large number of tests will allow to achieve high precision of calculations. At an initial stage the choice of casual parameters was made with uniform distribution in the field of admissible values, and then were specified with normal distribution of a random variable. At the same time the range of change of geometrical parameters of a tooth zone was in the following limits: from 0 to 10 $\mathrm{mm}$ - tooth crown width range; from 8 to $15 \mathrm{~mm}$ - tooth width range; from 0 to $4 \mathrm{~mm}-$ tooth crown height range. At the same time current of a phase changed in the range from 10 to 30 And.

For determination of numerical value of criterion function, it is necessary to carry out electromagnetic calculation. On the basis of the carried-out analysis of implementers of mathematical model of the main software packages [10] allowing to carry out calculation of electromagnetic devices (ELCUT, FEMM, GMSH+GetDP) as a programming environment for performance of computing experiments with the electromechanical converter with a discrete secondary part the software package of Ansys Maxwell has been chosen Ansys.

Upon transition directly to the solution of an optimizing task, it is necessary to set initial values of geometrical parameters of a tooth zone of the inductor which search of optimum values is carried out. At this stage, the initial geometrical sizes of a tooth zone received based on the carried-out earlier analytical calculation. At the same time external and internal diameters of the inductor by optimization don't change.

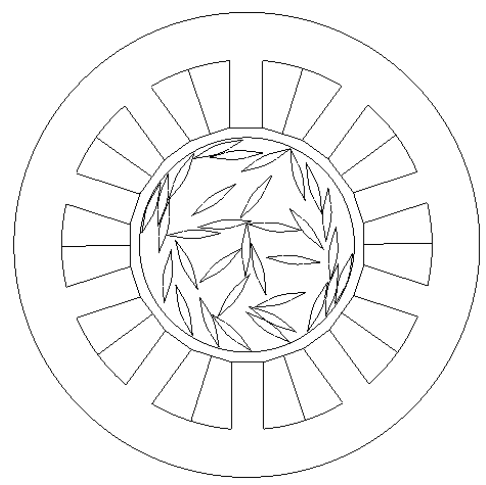

Fig. 3. Settlement area for the analysis of the electromagnetic field.

At creation of settlement model were assumption according to which the arrangement of ferromagnetic elements is chaotic and chaotic, and fixed for all experiments is accepted. In ECDSP the electromagnetic field exists in the following set of environments: ferromagnetic core of the inductor (brand 2214 steel); the processed liquid environment (when calculating has been replaced with air); the areas occupied with conductors with current; ferromagnetic elements.

Boundary conditions for vector magnetic potential on external borders of settlement areas of geometrical model were set proceeding from field living conditions on these borders and the accepted assumptions. On external border of settlement area of the inductor of the electromechanical converter with a discrete secondary part the known assumption of $\mathrm{A}=0[12]$ is accepted. 
After creation of geometrical model the grid of final elements presented in fig. 4 is automatically formed.

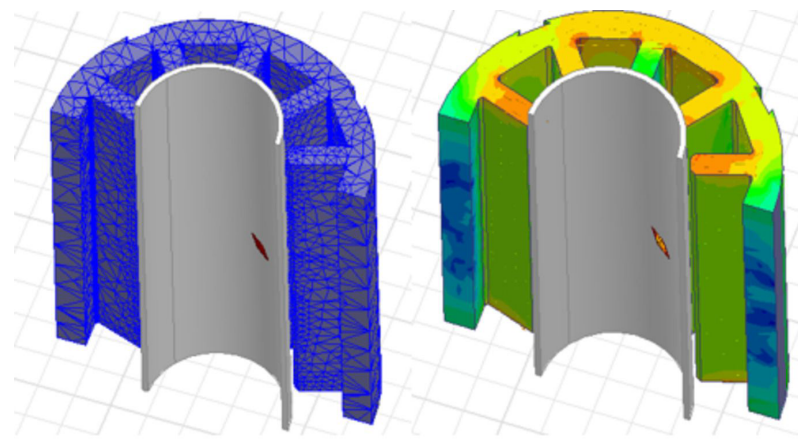

Fig. 4. Grid of final elements.

\section{Results}

As a result of carrying out parametrical optimization of the inductor of the electromechanical converter on complex criterion function various results have been received. When using in criterion function only of the first composed, average value of magnetic induction in volume of the working camera, the equal $0,1 \mathrm{~T}$, have been received unacceptably high value of phase current equal $20 \mathrm{~A}$, and, therefore, the condition on heating of a magnetic conductor wasn't satisfied. However, as it was already stated above, performance only of a condition of ensuring necessary average value of magnetic induction in volume of the working camera, can't lead to effective operation of the device when there is "sticking" of elements in the field of corners of teeth of the stator, owing to repeated excess of values of magnetic induction. If the optimizing problem is solved along with accounting of all components of criterion function, the optimum average value of electromagnetic effort on the volume of the working camera is reached at rather high, for this type of devices, average value of magnetic induction in volume of the working camera, equal 0,07 $\mathrm{T}$ and at rather low value of phase current $14 \mathrm{~A}$.

The carried-out optimizing calculations have confirmed the hypothesis received on the basis of theoretical researches of various configurations of a tooth zone that it is possible to increase value of the electromagnetic effort operating on a single ferromagnetic element from the electromagnetic field at optimum selection of the geometrical sizes of a tooth, including the geometrical sizes of a crown of a tooth. The sketch of geometry of the tooth zone received by results of parametrical optimization is submitted in fig. 5 .

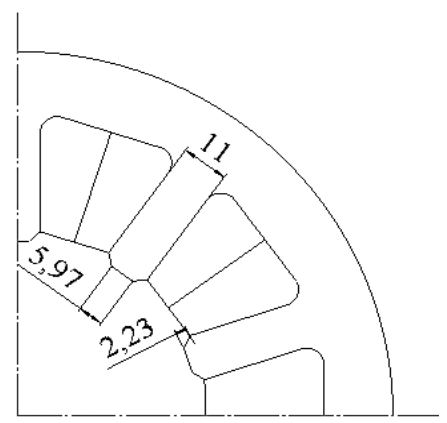

Fig. 5. Section of a tooth zone of the optimized inductor design. 
For the purpose of demonstration of efficiency of optimizing calculation, it has been carried out, on the basis of results of electromagnetic calculation in a software package of Ansys, comparison of an initial configuration with optimized. Comparative results are presented in tab. 2. As an estimated indicator values of electromagnetic effort for a ferromagnetic element at various distances from an active pole have been chosen.

\section{Conclusions}

Application of multicriteria optimization allows to improve ECDSP indicators that it has been shown on the example of design of the electromechanical converter on the basis of the 10th phase inductor.

As show results of the calculations which are carried out for two options of designs of the inductor ECDSP, increase in the electromagnetic effort operating on a single ferromagnetic element has reached on average in volume to the working camera $29 \%$, at the same value of phase current. Achievement of this indicator is more important for cases of work with the elements most remote from an active pole, that is being in area of a "dead" zone of the 2 nd order, in the center of the working camera.

\section{References}

1. T.V. Kirbyatyeva, L.P. Kortovenko, I.E. Mordvinova, Inzhenerno-stroitel'nyy vestnik Prikaspiya, 3, 17 (2013)

2. G.I. Volodin, Electromagnetic processes in devices with any mobile part: yew. Dr.Sci.Tech.: 05.09.01 (Novocherkassk, 2009)

3. N.A. Derevyakin, Z.A. Mikhaleva, Devices with a vortex layer in chemical technology. Survey information. Chemical and oil processing mechanical engineering. HM-1 series. (Tambov institute of chemical mechanical engineering, Tambov, 1989)

4. D.N. Kuimov, M.S. Minkin, A.D. Lukyanov, Materials Science Forum, 870, 671 (2016)

5. M.S. Minkin, D.N. Kuimov, M.N. Kulinich, In the collection: Dynamics of the technical Collection of Works XII of the International Scientific and Technical Conference systems, 392 (2016)

6. D.N. Kuimov, M.S. Minkin, MATEC Web Conf, 132, 03016 (2017)

7. D.N. Kuimov, M.S. Minkin, Patent RU169608 (2016)

8. A.V. Shevkunova, New science: Problems and prospects, 3-2, 248 (2016)

9. A.V. Kashuba, Works of the Rostov state transport university, 3, 62 (2016)

10. D.A. Shchuchkin, A.V. Pavlenko, V.S. Puzin, D.V. Batishchev, A.S. Horoshev, Electrical equipment, 12, 88 (2017)

11. N.N. Klochkova, A.V. Obukhova, Series: Technical science, 2, 161 (2010)

12. E.A. Klimov, V.V. Kolosov, V.E. Saprykin, News of higher educational institutions. Electromecanics, Novocherkassk, 1, 67 (2012)

13. V. A. Tyukov, V.V. Pastukhov, K.V. Korneev, TPU News, 4, 99 (2011) 